\title{
Left Ventricular Remodeling of Hypertrophic Cardiomyopathy Longitudinal Observation in a Rural Community
}

\author{
Hiroaki Kitaoka, MD; Toru Kubo, MD; Makoto Okawa, MD; Nobuhiko Hitomi, MD; \\ Takashi Furuno, MD; Yoshinori L Doi, MD
}

\begin{abstract}
Background The purpose of the present study was to assess the clinical long-term course of hypertrophic cardiomyopathy (HCM) in a rural Japanese cohort.

Methods and Results A total of 137 consecutive HCM patients (mean age at diagnosis: $52 \pm 13$ years) were enrolled. During a follow-up period of 11.4 \pm 5.7 years, 28 patients died of HCM-related causes. Eleven patients died suddenly, 10 died of progressive heart failure, 6 died of stroke associated with atrial fibrillation and 1 died of a postoperative complication of alcohol septal ablation. For the overall study group, 5-, 10- and 15-year cumulative survival rates were $91 \%, 88 \%$ and $79 \%$, respectively. Although sudden death was the predominant cause of HCM-related death during the follow-up period of $<10$ years, heart failure death increased after follow-up period of $>10$ years. Fifteen (13\%) of 114 patients who had follow-up echocardiography became 'end-stage' HCM and 8 patients died of severe and refractory heart failure. They already showed minimally dilated left ventricular (LV) dimension and lower LV fractional shortening at initial evaluation.

Conclusions Although HCM patients in a Japanese rural community showed relatively benign clinical course (the same as cohorts in the developed world), heart failure death because of LV remodeling became equally important to sudden death when they were followed for more than 10 years. (Circ J 2006; 70: 1543-1549)
\end{abstract}

Key Words: Heart failure; Hypertrophic cardiomyopathy; Prognosis

$\mathbf{H}$ ypertrophic cardiomyopathy ( $\mathrm{HCM})$ is a primary myocardial disease that has been defined as a left ventricular (LV) hypertrophy without other cardiovascular diseases and has heterogeneous clinical and morphologic features! ${ }^{1-4}$ The natural history of HCM varies from an asymptomatic and benign clinical course to sudden premature death. A high annual mortality rate of $3-4 \%$ was reported from major referral centers ${ }^{5-8}$ Recent observations in community-based cohorts in the USA and Europe suggest a more favorable prognosis compared with the results of previous studies?-13

Because of lifelong LV remodeling, clinical and morphologic presentations could well be changed in HCM in long-term observation! ${ }^{14-20}$ Moreover, there is little longterm prognostic information in older HCM patients living in a rural community. The purpose of the present study was therefore to assess the long-term prognosis in the older rural HCM patients.

\section{Methods}

\section{Patient Population}

Kochi Medical School Hospital is a community-based hospital primarily supporting the Kochi prefecture (population of 810,000) in the southwest part of Japan. Between 1983 and June 2001, 137 consecutive patients with HCM (95 males and 42 females; age range, 9 to 79 years; mean

(Received June 20, 2006; revised manuscript received September 25, 2006; accepted October 4, 2006)

Cardiology Division, Department of Medicine and Geriatrics, Kochi Medical School, Kochi, Japan

Mailing address: Yoshinori L Doi, MD, Department of Medicine and Geriatrics, Kochi Medical School, Oko-cho, Nankoku-shi, Kochi 783-8505, Japan. E-mail: ydoi@med.kochi-u.ac.jp age, $52 \pm 13$ years) were retrospectively assessed at our hospital. All patients were assessed for the clinical, electrocardiographic and echocardiographic findings at their first visit. Holter electrocardiogram monitoring was also assessed, if possible at their first visit $(\mathrm{n}=103)$, or during follow-up if arrhythmogenic event was suspected. As a result, 117 patients were assessed by Holter monitoring at least once during follow-up. The study protocol was approved by the Ethics Committee of our hospital.

\section{Echocardiography and Definitions}

Echocardiographic measurements were performed with a commercially available Toshiba instrument (Tokyo, Japan) using $3.5 \mathrm{MHz} / 2.75 \mathrm{MHz}$ transducers. The dimensions of the left ventricle and the left atrium were measured according to previously published criteria. ${ }^{21}$ The magnitude and distribution of LV hypertrophy was also assessed using 2dimensional images and indices as previously described? 1 To define the magnitude of LV hypertrophy, the left ventricle was divided into 4 equal segments (anterior septum, posterior septum, lateral wall and posterior wall) at the level of the mitral valve and papillary muscles, and 1 segment at the apical level 22 The greatest wall thickness measured at any site in the left ventricle was regarded as maximal thickness. Intraventricular pressure gradient was also measured using continuous-wave Doppler under basal conditions. The LV outflow gradient $\geq 30 \mathrm{mmHg}$ was considered significant.

The diagnosis of HCM was based on the echocardiographic demonstration of a hypertrophied left ventricle (wall thickness $\geq 15 \mathrm{~mm}$ ) without hypertension and other cardiac diseases, capable of producing the magnitude of wall thickening evident ${ }^{23}$ Apical HCM was defined as LV wall thickening confined to the most distal region of the LV 
Table 1 Clinical Characteristics of the Patients With HCM

\begin{tabular}{lcccc}
\hline \hline & $\begin{array}{c}\text { Survivors } \\
(n=109)\end{array}$ & $\begin{array}{c}\text { Non-survivors } \\
(n=28)\end{array}$ & $\begin{array}{c}\text { Sudden death } \\
(n=11)\end{array}$ & $\begin{array}{c}\text { Heart failure death } \\
(n=10)\end{array}$ \\
\hline Male, gender, $n(\%)$ & $79(72)$ & $16(57)$ & $5(45)$ & $7(70)$ \\
Follow-up (years) & $12 \pm 5$ & $9 \pm 7^{*}$ & $7 \pm 7^{\#}$ & $11 \pm 7$ \\
Age at diagnosis (years) & $52 \pm 13$ & $55 \pm 13$ & $54 \pm 19$ & $54 \pm 10$ \\
Age at HCM-related death (years) & & $64 \pm 14$ & $62 \pm 20$ & $63 \pm 10$ \\
NYHA functional class & $101(93)$ & $21(75)^{\#}$ & $10(91)$ & $5(50)^{\dagger}$ \\
I/II, $n(\%)$ & $8(8)$ & $7(25)$ & $1(9)$ & $5(50)$ \\
III/IV, $n(\%)$ & $43(39)$ & $16(57)$ & $4(36)$ & $7(70)$ \\
FH of HCM, $n(\%)$ & $25(23)$ & $11(39)$ & $3(27)$ & $5(50)$ \\
FH of sudden death, $n(\%)$ & $12(11)$ & $2(7)$ & $1(9)$ & $0(0)$ \\
History of syncope, $n(\%)$ & $31(28)$ & $20(71)^{\dagger}$ & $6(55)$ & $7(70)^{\sharp}$ \\
Atrial fibrillation & $9(8)$ & $13(46)$ & $3(27)$ & $4(40)$ \\
Overall, $n(\%)$ & $22(20)$ & $7(25)$ & $3(27)$ & $3(30)$ \\
At initial evaluation, $n(\%)$ & $23(26)$ & $13(46)^{\#}$ & $2(18)$ & $8(80)^{\dagger}$ \\
During follow-up, $n(\%)$ & & & \\
Non-sustained VT, $n(\%)$ & & & \\
\hline
\end{tabular}

${ }^{\dagger} p<0.001,{ }^{*} p<0.005,{ }^{*} p<0.01 ;$ compared with survivors.

HCM, hypertrophic cardiomyopathy; NYHA, New York Heart Association; FH, family history; VT, ventricular tachycardia.

Table 2 Echocardiographic Findings at First Evaluation of the Patients With HCM

\begin{tabular}{|c|c|c|c|c|}
\hline & $\begin{array}{c}\text { Survivors } \\
(n=109)\end{array}$ & $\begin{array}{c}\text { Non-survivors } \\
\quad(n=28)\end{array}$ & $\begin{array}{l}\text { Sudden death } \\
\quad(n=11)\end{array}$ & $\begin{array}{l}\text { Heart failure death } \\
\qquad(n=10)\end{array}$ \\
\hline LV end-diastolic dimension ( $\mathrm{mm}$ ) & $44 \pm 6$ & $45 \pm 5$ & $42 \pm 8$ & $49 \pm 5^{+}$ \\
\hline LV end-systolic dimension (mm) & $27 \pm 6$ & $29 \pm 7$ & $24 \pm 4$ & $33 \pm 8^{\#}$ \\
\hline IVS thickness (mm) & $17 \pm 5$ & $18 \pm 3$ & $18 \pm 7$ & $18 \pm 2$ \\
\hline LV posterior wall thickness ( $\mathrm{mm}$ ) & $11 \pm 7$ & $11 \pm 3$ & $11 \pm 4$ & $12 \pm 2$ \\
\hline Left atrial dimension ( $\mathrm{mm}$ ) & $38 \pm 7$ & $42 \pm 7^{\#}$ & $38 \pm 6$ & $45 \pm 7 *$ \\
\hline Maximal wall thickness (mm) & $20 \pm 4$ & $20 \pm 4$ & $22 \pm 5$ & $18 \pm 2$ \\
\hline Fractional shortening (\%) & $40 \pm 9$ & $37 \pm 11$ & $43 \pm 7$ & $33 \pm 10^{+}$ \\
\hline LV outflow gradient, $n(\%)$ & $15(14)$ & $4(14)$ & $3(27)$ & $0(0)$ \\
\hline
\end{tabular}

${ }^{*} p<0.005,{ }^{*} p<0.01,{ }^{*} p<0.05$; compared with survivors.

Data are mean $\pm S D$.

LV, left ventricular; IVS, interventricular septum. Other abbreviations see in Table 1.

apex below the papillary muscle level, non-detectable in parasternal echocardiographic cross-sectional planes but detectable in apical views ${ }^{23}$ The LV systolic dysfunction was defined as percentage fractional shortening $<25 \%$. Concomitant coronary artery disease was excluded either by coronary angiography and/or myocardial scintigraphy.

\section{Follow-up and Endpoints}

Initial clinical investigation was defined as the time when the diagnosis of HCM was first confirmed at our hospital. Follow-up was carried out using available medical records and telephone interviews with the patients and/or referring physicians. The cardiovascular death as a result of 3 specified endpoints was assessed. The first endpoint was sudden death; unexpected sudden collapse or nocturnal death in patients with a relatively uneventful previous clinical course. In addition, patients with successful resuscitation from ventricular fibrillation were included as a sudden death in the present study. The second endpoint was heart failure death; occurring in the context of cardiac decompensation and progressive disease course $\geq 1$ year before death, particularly if complicated by pulmonary edema or evolution to the end-stage phase and/or requiring hospitalization for heart-failure. Sudden death and appropriate discharge of an implantable cardioverter-defibrillator (ICD) in the patients with severe heart failure symptoms were also included to heart failure death. The third endpoint was stroke death; judged to be a direct consequence of embolic or other events related to HCM, usually in the setting of paroxysmal or chronic atrial fibrillation, documented by computed tomography.

\section{Therapeutic Strategies}

Patients without LV systolic dysfunction were treated with a $\beta$-blocker or calcium antagonist. Patients with LV outflow gradient were also treated with a $\beta$-blocker. When the reduction of gradient was insufficient, a calcium antagonist or $\mathrm{Na}$ channel blocker (dysopiramide or cibenzoline) were added in addition to a $\beta$-blocker. If LV systolic dysfunction was observed, treatment with a $\beta$-blocker or a calcium antagonist was discontinued, and diuretics, angiotensin-converting enzyme inhibitor/angiotensin receptor blocker and digitalis were given according to a guideline for management of chronic heart failure.

\section{Statistical Analysis}

Values were presented as means \pm SD. Differences between means were analyzed using unpaired Student's t-test and comparison of nominal variables expressed as proportions was calculated using the chi-square test. Analysis of event-free curves was calculated using Kaplan-Meier estimates and comparison of curves was done by the logrank test. A p-value $<0.05$ was considered statistically significant. All calculations were performed using Statview ver.5.0 (SAS Institute, Inc, Cary, NC, USA). 


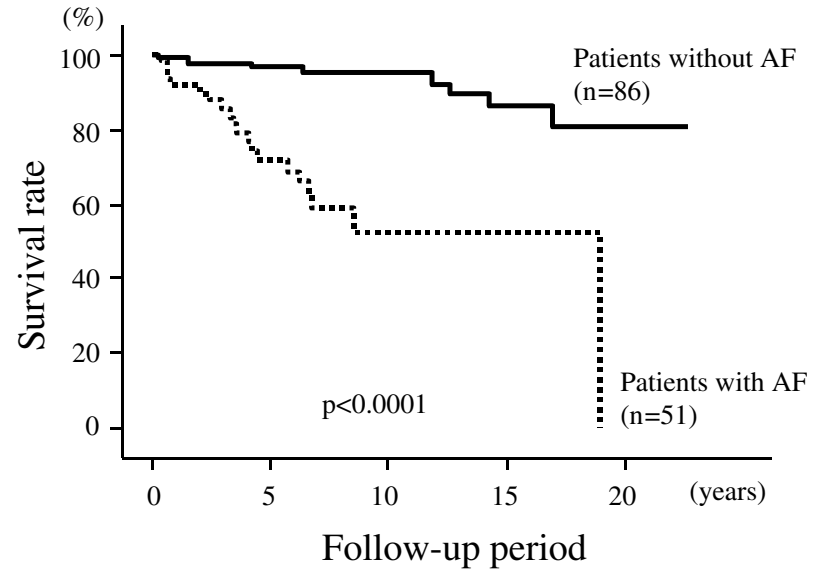

Fig 1. Survival curves of patients with and without atrial fibrillation (AF). The survival rate for patients with $\mathrm{AF}$ was worse than for those without.

\section{Results}

\section{Clinical Characteristics}

The age at diagnosis of all patients was $52 \pm 13$ years and 95 patients $(68 \%)$ were male. Nine patients $(6 \%)$ were younger than 30 years and 47 patients $(34 \%)$ were 60 years or older. One hundred twenty-two patients $(89 \%)$ had no or only mild cardiac symptoms (New York Heart Association functional class I/II) at initial evaluation. Fifty-nine patients $(43 \%)$ were thought to have familial HCM and 36 patients $(26 \%)$ had a family history of sudden death. At entry of the study, 47 patients were receiving $\beta$-blocker and 38 patients were receiving calcium antagonist. A combination of $\beta$-blocker and calcium antagonist was given in 9 patients and a combination of $\beta$-blocker and Na channel blocker in 2 patients. During follow-up, 6 patients (4\%) received amiodarone (usually $100 \mathrm{mg} /$ day). Four patients $(3 \%)$ received ICD, 1 patient received pacemaker implantation for LV outflow gradient and 1 patient underwent alcohol septal ablation. No patients received surgical treatment for LV outflow gradient.

Twenty-two patients (16\%) had paroxysmal or persistent atrial fibrillation at the initial assessment. During follow-up of 5.5 \pm 6.4 years from the initial evaluation, paroxysmal or persistent atrial fibrillation was newly documented by standard electrocardiogram or Holter monitoring in 29 patients. Thus, 51 patients $(37 \%)$ were complicated by atrial fibrillation. Non-sustained ventricular tachycardia (defined as $>3$ beats) was documented in 36 of 117 patients $(31 \%)$.

\section{Initial Echocardiographic Findings}

Maximal LV wall thickness determined by echocardiography was $20 \pm 4 \mathrm{~mm}$ (range: 15 to $33 \mathrm{~mm}$ ). Ninety-six patients $(73 \%)$ showed a diffuse distribution of hypertrophy in more than 2 segments of the left ventricle at the level of the mitral valve. Thirty-seven patients $(27 \%)$, including 22 patients (16\%) with apical HCM, had hypertrophy in 1 segment of the left ventricle. Four patients (3\%) showed reduced LV systolic function, defined as fractional shortening of $<25 \%$, at diagnosis. Resting LV outflow gradient $(\geq 30 \mathrm{mmHg})$ was present in 19 of the 137 patients (14\%), ranging from $31 \mathrm{mmHg}$ to $140 \mathrm{mmHg}$. Another 2 patients had mid-ventricular obstruction.

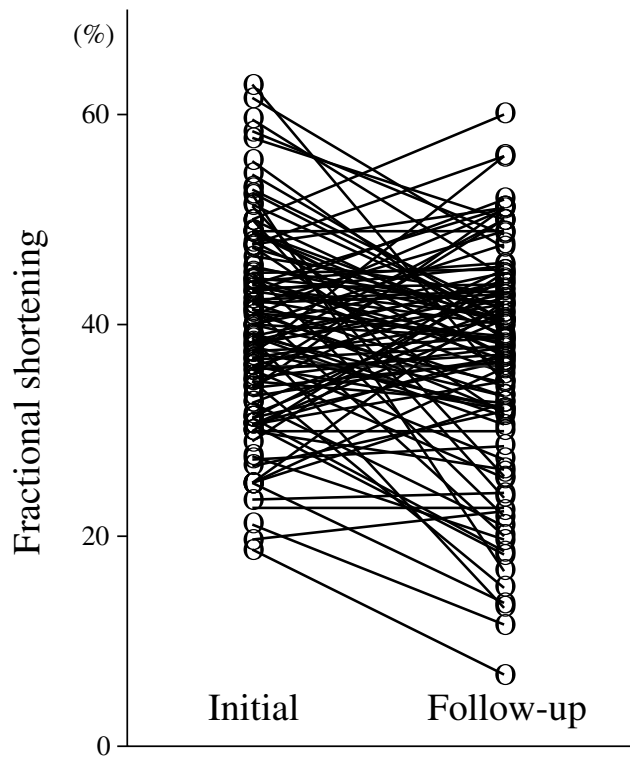

Fig 2. Change in $\%$ fractional shortening of the left ventricle during follow-up period of $9.4 \pm 4.9$ years. Most patients show no significant change of left ventricular systolic function, but some have decreased function.

\section{Mortality and Prognostic Predictors in HCM-Related Death}

During follow-up of $11.4 \pm 5.7$ years, 28 of the 137 patients died of causes directly related to HCM. Eleven patients died suddenly and unexpectedly, 10 died of heart failure, 6 died of embolic stroke associated with atrial fibrillation and 1 died of a postoperative complication of percutaneous alcohol septal ablation. For the overall study group, 5-, 10- and 15-year cumulative survival rates were $91 \%, 88 \%$ and $79 \%$, respectively.

The clinical and initial echocardiographic features comparing survivors and non-survivors were shown in Table 1 and Table 2. Non-survivors had more severe symptoms at initial assessment, higher prevalence of atrial fibrillation and non-sustained ventricular tachycardia than survivors. Fig 1 showed the survival curve of patients with atrial fibrillation and that of those without atrial fibrillation. The prognosis of patients with atrial fibrillation was worse than that of those without atrial fibrillation. For the patients with atrial fibrillation, 1-, 3- and 5-years cumulative survival rate from the day of first documentation of atrial fibrillation were $92 \%, 86 \%$ and $72 \%$, respectively. By echocardiography, non-survivors showed larger left atrial dimension compared to survivors and none had apical HCM.

Moreover, clinical and echocardiographic features were also compared between survivors and patients with sudden death/heart failure death (Tables 1,2). Patients with sudden death showed no significant difference in clinical and echocardiographic features compared with survivors in our cohort. However, patients with heart failure death showed severe symptoms at initial assessment, higher prevalence of atrial fibrillation and non-sustained ventricular tachycardia during follow-up. Moreover, patients with heart failure death showed larger LV dimension and lower LV fractional shortening at initial assessment compared with the survivors.

The mode of death was shown in Fig 2. Sudden death was a predominant cause of HCM-related death during 


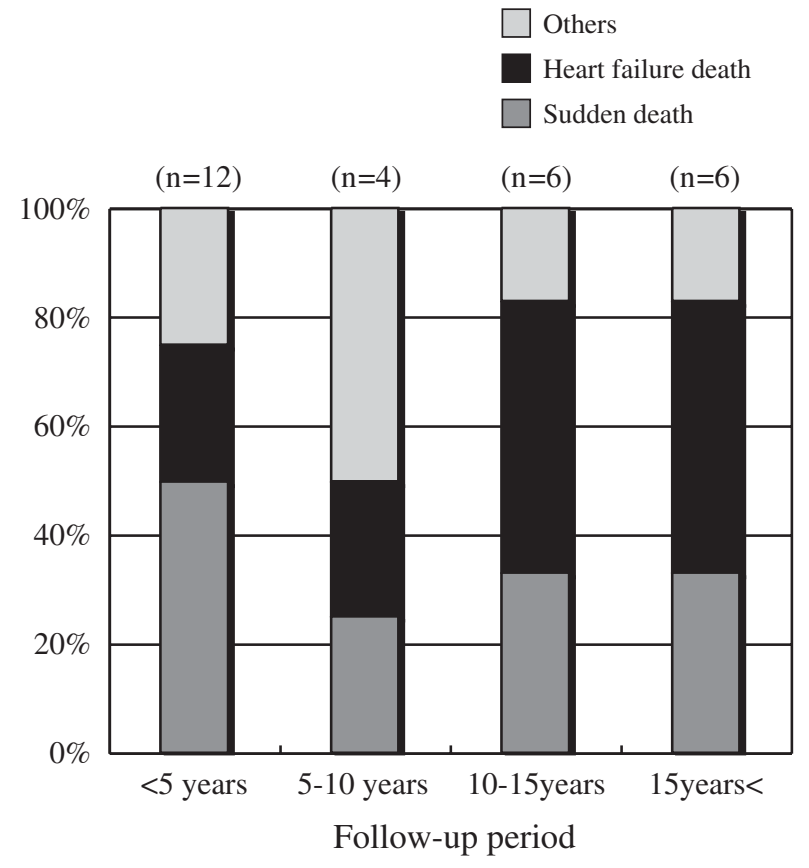

Fig 3. Cause of death during long-term follow-up. Although sudden death was the predominant cause of hypertrophic cardiomyopathyrelated death during follow-up period of $<10$ years, heart failure death became predominant after a follow-up period of $>10$ years.

relatively short-term period. However, heart failure death became predominant after a follow-up period of $>10$ years. Although $6(50 \%)$ of the 12 twelve patients who died during follow-up period of $<5$ years died suddenly, 6 (50\%) of the 12 patients who died during follow-up period of $>10$ years died of heart failure.

\section{Remodeling During Follow-up}

Follow-up echocardiographic data during the interval of $9.4 \pm 4.9$ years were obtained from 114 patients. The LV end-diastolic dimension significantly increased from $44.5 \pm 5.9 \mathrm{~mm}$ to $46.9 \pm 7.6 \mathrm{~mm}(\mathrm{p}<0.0001)$, and LV endsystolic dimension also increased from $27.2 \pm 6.8 \mathrm{~mm}$ to $30 \pm 9.3 \mathrm{~mm}(\mathrm{p}=0.0001)$. The change of fractional shorten- ing in 114 patients was shown in Fig 3. Fractional shortening significantly decreased from $39.7 \pm 9.5 \%$ to $37 \pm 10.2 \%$ $(\mathrm{p}=0.02)$. In addition to 4 patients with impaired LV systolic function at initial assessment, 11 patients evolved from preserved LV systolic function into impaired LV systolic function during follow-up. Thus, 15 patients (13\%) among 114 patients showed the 'end-stage' HCM and 8 patients died of severe and refractory heart failure. Tables 3 and 4 showed the comparisons of clinical and echocardiographic findings between patients with systolic dysfunction and those without LV systolic dysfunction. Patients with LV systolic dysfunction (even 11 patients evolved from normal LV systolic function into LV systolic dysfunction) had more severe symptoms, complication of atrial fibrillation, larger LV dimensions and lower LV fractional shortening at initial assessment compared with those without LV systolic dysfunction.

\section{Discussion}

Although older HCM patients in a rural Japanese community showed relatively benign clinical course and the same as the previous reports in community-based western HCM cohort, the present study indicated that heart failure death was equally important to sudden death in older HCM patients particularly after long-term follow-up, because a significant part (11\%) of our patients evolved into LV systolic dysfunction.

\section{Prognosis of HCM Patients}

Because the initial clinical description of HCM in the early 1960 s, most information on this disease has been reported from large referral centers 5,6 It is likely that the most severely affected patients are referred to such tertiary centers. Recent observations in community-based cohorts in the USA and Europe suggest much lower mortality rates in patients with HCM9-13 Maron et al reported that 29 deaths out of 277 patients (age at diagnosis: $47 \pm 22$ years) with HCM in a regional cohort in the USA were directly related to HCM during a mean follow-up period of $8.1 \pm$ 6.6 years? The annual HCM mortality rate was $1.3 \%$, and the 5-, 10- and 15-year cumulative survival rates were $93.5 \%, 89.5 \%$ and $83.2 \%$, respectively9 Cecchi et al reported the result of the Italian nationwide survey of HCM,

Table 3 Clinical Findings of the Patients With LV Systolic Dysfunction

\begin{tabular}{lccc}
\hline \hline & Group $A(n=15)$ & Group $B(n=11)$ & Group $C(n=99)$ \\
\hline Male, gender, $n(\%)$ & $8(53)$ & $6(55)$ & $78(68)$ \\
Follow-up (years) & $14 \pm 7$ & $14 \pm 6$ & $12 \pm 5$ \\
Age at diagnosis (years) & $51 \pm 14$ & $50 \pm 16$ & $52 \pm 13$ \\
NYHA functional class & & & \\
I/II, $n(\%)$ & $8(53)^{\dagger}$ & $6(55)^{+}$ & $92(93)$ \\
III/IV, $n(\%)$ & $7(47)$ & $5(45)$ & $7(7)$ \\
FH of HCM, $n(\%)$ & $9(60)$ & $6(55)$ & $38(38)$ \\
FH of sudden death, $n(\%)$ & $5(33)$ & $3(27)$ & $23(23)$ \\
History of syncope, $n(\%)$ & $1(6)$ & $0(0)$ & $13(13)$ \\
Atrial fibrillation & $12(80)^{\dagger}$ & $9(82)^{*}$ & $31(31)$ \\
Overall, $n(\%)$ & $4(27)$ & $2(18)$ & $10(10)$ \\
At initial evaluation, $n(\%)$ & $8(53)$ & $7(64)$ & $21(21)$ \\
During follow-up, $n(\%)$ & $10(67)^{*}$ & $7(64)^{*}$ & $21(21)$ \\
Non-sustained VT, $n(\%)$ & & & \\
\hline
\end{tabular}

Group A, all patients with LV systolic dysfunction; Group B, patients developed LV systolic dysfunction during follow-up; Group C, patients without $L V$ systolic dysfunction.

${ }^{t} p<0.001, * p<0.005,{ }^{*} p<0.05$; compared with Group $C$.

Abbreviations see in Tables 1,2. 
Table 4 Echocardiographic Findings of the Patients With LV Systolic Dysfunction

\begin{tabular}{lccc}
\hline \hline & Group A $(n=15)$ & Group B $(n=11)$ & Group C $(n=99)$ \\
\hline Interval of echocardiography (years) & $11 \pm 5$ & $11 \pm 6$ & $9 \pm 5$ \\
At initial evaluation & & & \\
LV end-diastolic dimension (mm) & $51 \pm 8^{\dagger}$ & $49 \pm 5^{*}$ & $44 \pm 6$ \\
LV end-systolic dimension (mm) & $36 \pm 8^{\dagger}$ & $34 \pm 7^{\dagger}$ & $26 \pm 6$ \\
IVS thickness (mm) & $18 \pm 5$ & $20 \pm 5$ & $17 \pm 5$ \\
LV posterior wall thickness (mm) & $11 \pm 2$ & $11 \pm 2$ & $11 \pm 3$ \\
Left atrial dimension $(\mathrm{mm})$ & $44 \pm 6^{\dagger}$ & $42 \pm 5^{\dagger}$ & $38 \pm 6$ \\
Maximal wall thickness (mm) & $18 \pm 5$ & $20 \pm 5$ & $20 \pm 4$ \\
Fractional shortening (\%) & $30 \pm 10^{\dagger}$ & $32 \pm 10^{*}$ & $41 \pm 9$ \\
LV outflow gradient, $n(\%)$ & $0(0)$ & $0(0)$ & $19(19)$ \\
At last evaluation & $60 \pm 6^{\dagger}$ & $58 \pm 5^{\dagger}$ & $45 \pm 6$ \\
LV end-diastolic dimension (mm) & $49 \pm 7^{\dagger}$ & $46 \pm 5^{\dagger}$ & $27 \pm 5$ \\
LV end-systolic dimension (mm) & $13 \pm 3^{*}$ & $14 \pm 3^{\dagger}$ & $17 \pm 5$ \\
IVS thickness (mm) & $10 \pm 2^{\dagger}$ & $10 \pm 1$ & $11 \pm 2$ \\
LV posterior wall thickness $(\mathrm{mm})$ & $53 \pm 8^{\dagger}$ & $53 \pm 7^{*}$ & $44 \pm 8$ \\
Left atrial dimension $(\mathrm{mm})$ & $18 \pm 5^{\dagger}$ & $21 \pm 3^{\dagger}$ & $40 \pm 7$ \\
Fractional shortening $(\%)$ & & &
\end{tabular}

Group A, all patients with LV systolic dysfunction; Group B, patients developed LV systolic dysfunction during follow-up; Group C, patients without $L V$ systolic dysfunction.

All data except $L V$ outflow gradient are mean $\pm S D$.

${ }^{\dagger} p<0.001,{ }^{*} p<0.005,{ }^{*} p<0.05$; compared with Group $C$.

Abbreviations see in Table 2.

in which 1,677 patients $(44 \pm 19$ years $)$ were enrolled!2 During Cecchi and colleagues' follow-up period of $9.7 \pm$ 7.7 years, the annual cardiovascular death was $1.0 \%$.

The Kochi prefecture that our hospital supports is a rural part of Japan where older people are predominant. In 2001, $24.1 \%$ of people living in Kochi prefecture were older than 65 years. Accordingly, our patient cohort (age at diagnosis: $52 \pm 13$ years) was older than previous reports and followup period was longer (11.4 \pm 5.7 years) $)^{9-13,24}$ because the movement of peoples was less in such a rural community. The results of the present study in older HCM cohort showing favorable prognosis are consistent with the reports on HCM in community-based populations in the USA and Italy. This result adds further evidence to confirm our recent understanding that HCM dose not necessarily represent a generally unfavorable disease.

\section{Cause of Death and LV Remodeling in HCM Patients}

In the past 2 decades, much attention has been given to the prediction of sudden and unexpected death, which was reported to be the most common and unfortunate cause of death in patients with $\mathrm{HCM}^{1-8,25,26}$ However, a significant portion of patients with HCM in a regional cohort population dies from progressive heart failure and stroke as a result of atrial fibrillation, in addition to premature sudden death!12,27 In particular, heart failure death is usually caused by LV systolic dysfunction secondary to LV remodeling! ${ }^{1-18}$ Although the precise mechanism of this remodeling is unresolved, coronary microvascular ischemia is suggested as one of the important mechanisms. ${ }^{19}$ This LV remodeling is found in about $5-10 \%$ of adult HCM patients, but the longer the follow-up is, the higher the prevalence of LV remodeling is $!^{16-18}$ In fact, $13 \%$ of our patients, who had a follow-up echocardiographic date, evolved into LV remodeling during long-term follow-up and patients with LV remodeling showed severely deteriorated clinical presentation and poor prognosis.

Although several factors (family history of sudden death, history of syncope, non-sustained ventricular tachycardia, abnormal blood pressure response during exercise, extreme LV hypertrophy and some abnormal sarcomeric gene mutations) were proposed for the predictor of sudden death, $-5,25,26$ the prediction of heart failure death because of LV remodeling is difficult, because of the absence of precise predictive factors $14-20$ In the present study, patients who evolved into LV systolic dysfunction already had larger LV dimensions and lower LV fractional shortening (although such parameters were within the 'normal' range, they were rather larger for HCM) at initial assessment. This means that progression to LV systolic dysfunction might possibly be detected by careful investigation of the echocardiographic date.

The clinical and prognostic significance of diastolic function derived from Doppler echocardiographic parameters is unresolved in HCM. Although the degree of diastolic dysfunction assessed by Doppler echocardiography was not assessed in the current study because of retrospective design of the study, several investigators reported the lack of significant relation between the symptoms/exercise capacity and diastolic function derived from transmitral flow at rest (peak flow velocity in early diastole (E), and during atrial filling (A), the E/A ratio) because of a wide range of pattern ${ }^{28,29}$ Therefore, conventional Doppler indexes provide little clinical and prognostic information. However, new parameters such as myocardial velocity gradient and mitral annular velocities assessed using tissue Doppler imaging, might be useful in non-invasively and precisely assessing the degree of diastolic dysfunction in patients with HCM 30,31 Recently, we reported that myocardial performance index (TEI index), that reflected both systolic and diastolic functions, was abnormal and correlated with plasma B-type natriuretic peptide level in patients with $\mathrm{HCM}^{22}$ Thus, those newly developed echocardiographic parameters in patients with HCM might add the more clinical and prognostic information. Further studies are needed.

Another significant predictive factor was complication of atrial fibrillation. Atrial fibrillation is a common and important arrhythmia in $\mathrm{HCM}^{32}$ Atrial fibrillation is associated with substantial risk for heart failure death, stroke and severe functional disability and it has been reported that 
atrial fibrillation develops in $15-46 \%$ of patients with HCM. In fact, $37 \%$ of our patients complicated atrial fibrillation and higher prevalence of atrial fibrillation at initial assessment was observed in patients with heart failure death. In addition, about one-fourth of patients with atrial fibrillation died within 5 years from the day at first documentation of atrial fibrillation. Accordingly, patients with minimally dilated LV dimension, impaired LV systolic function for HCM at first evaluation and/or atrial fibrillation should be closely observed for the clinical and echocardiographic deterioration.

\section{Study Limitations}

First, the cohort size was relatively small and the design was retrospective. Second, our patient cohort differs from the other reports. Patients were older and had less prevalence of LV outflow gradient. Moreover, this difference might be affected by genetic background, because mutations in cardiac myosin binding-protein $\mathrm{C}$ gene were more common than those in the $\beta$-myosin heavy chain gene in our cohort 20

Third, there were no significant difference in previously reported prognostic risk factors of sudden death, such as family history of sudden death, history of syncope, nonsustained ventricular tachycardia and maximal wall thickness, between survivors and those who died suddenly. This might possibly be caused by patient selection (predominance of older patients without LV outflow gradient) and genetic background in our cohort ${ }^{24}$ Finally, our patient management could have affected the result of the current study. No patients received the surgical treatment for LV outflow gradient and only 2 patients underwent pacemaker implantation or alcohol septal ablation. In addition, no patients received ICD for the primary prevention of sudden death, because such treatment was not usual until recently in Japan.

\section{Conclusions}

Although older patients in a rural Japanese HCM cohort showed a relatively benign clinical course consistent with the HCM patients in the western world, heart-failure death should be recognized as an equally important mode of death in those living in a rural community when they were followed long enough for more than 10 years. Patients with minimally dilated LV dimension and/or impaired LV systolic function for HCM at initial assessment should be closely observed for the clinical and echocardiographic findings for longitudinal and continuous LV remodeling.

\section{References}

1. Maron BJ. Hypertrophic cardiomyopathy: A systematic review. JAMA 2002; 287: 1308-1320.

2. Maron BJ. Hypertrophic cardiomyopathy. Lancet 1997; 350: $127-$ 133.

3. Wigle ED, Rakowski H, Kimball BP, Williams WG. Hypertrophic cardiomyopathy: Clinical spectrum and treatment. Circulation 1995; 92: $1680-1692$.

4. Spirito P, Seidman CE, McKenna WJ, Maron BJ. The management of hypertrophic cardiomyopathy. N Engl J Med 1997; 336: $775-$ 785.

5. McKenna WJ, Deanfield JE, Faruqui A, England D, Oakley MC, Goodwin JF. Prognosis in hypertrophic cardiomyopathy: Role of age and clinical, electrocardiographic and hemodynamic features. Am J Cardiol 1981; 47: 532-538.

6. Maron BJ, Savage DD, Wolfson JK, Epstein SE. Progostic significance of 24 hour ambulatory electrocardiographic monitoring in pa- tients with hypertrophic cardiomyopathy: A prospective study. Am J Cardiol 1981; 48: 252-257.

7. Romeo F, Pelliccia F, Cristofani R, Martuscelli CE, Reale A. Hypertrophic cardiomyopathy: Is a left ventricular outflow tract gradient a major prognostic determinant? Eur Heart J 1990; 11: 233-240.

8. Seiler C, Hess OM, Schoenbeck M, Turina J, Jenni R, Turina M, et al. Long- term follow-up of medical versus surgical therapy for hypertrophic cardiomyopathy: A retrospective study. J Am Coll Cardiol 1991; 17: 634-642.

9. Maron BJ, Casey SA, Poliac LC, Gohman TE, Almquist AK, Aeppli DM. Clinical course of hypertrophic cardiomyopathy in a regional United States cohort. JAMA 1999; 281: 650-655.

10. Kofflard MJM, Ten Cate FJ, van der Lee C, van Domberg RT. Hypertrophic cardiomyopathy in a large community-based population: Clinical outcome and identification of risk factors for sudden cardiac death and clinical deterioration. J Am Coll Cardiol 2003; 41: $987-$ 993.

11. Cecchi F, Olivotto I, Montereggi A, Santoro G, Dolara A, Maron BJ. Hypertrophic cardiomyopathy in Tuscany: Clinical course and outcome in an unselected regional population. J Am Coll Cardiol 1995; 26: $1529-1536$.

12. Cecchi F, Olivotto I, Betocchi S, Rapezzi C, Conte MR, Sinagra G, et al. The Italian registry for hypertrophic cardiomyopathy: A nationwide survey. Am Heart J 2005; 150: 947-954.

13. Kyriakids M, Triposkiadis F, Anastasakis A, Theopistou A, Tocta R, Barbetseas J, et al. Hypertrophic cardiomyopathy in Greece. Chest 1998; 114: $1091-1096$.

14. Spirito P, Maron BJ, Bonow RO, Epstein SE. Occurrence and significance of progressive left ventricular wall thinning and relative cavity dilatation in hypertrophic cardiomyopathy. Am J Cardiol 1987; 60: $123-139$.

15. Maron BJ, Spirito P. Implications of left ventricular remodeling in hypertrophic cardiomyopathy. Am J Cardiol 1998; 81: 1339-1344.

16. Thaman R, Gimeno JR, Murphy RT, Kubo T, Sachdev B, Mogensen $\mathrm{J}$, et al. Prevalence and clinical significance of systolic impairment in hypertrophic cardiomyopathy. Heart 2005; 91: 920-925.

17. Biagini E, Coccolo F, Ferlito M, Perugini E, Rocchi G, Bacchireggiani L, et al. Dilated-hypokinetic evolution of hypertrophic cardiomyopathy. J Am Coll Cardiol 2005; 46: $1543-1550$.

18. Hina K, Kusachi S, Iwasaki K, Nogami K, Moritani H, Kita T, et al. Progression of left ventricular enlargement in patients with hypertrophic cardiomyopathy: Incidence and prognostic value. Clin Cardiol 1993; 16: 403-407.

19. Olivotto I, Cecchi F, Gistri R, Lorenzoni R, Chiriatti G, Girolami F, et al. Relevance of coronary microvascular flow impairment to longterm remodeling and systolic dysfunction in hypertrophic cardiomyopath. J Am Coll Cardiol 2006; 47: 1043-1048.

20. Kubo T, Kitaoka H, Okawa M, Matsumura Y, Hitomi N, Yamasaki $\mathrm{N}$, et al. Lifelong left ventricular remodeling of hypertrophic cardiomyopathy caused by a founder frameshift deletion mutation in the cardiac myosin-binding protein C gene among Japanese. J Am Coll Cardiol 2005; 46: $1737-1743$.

21. Maron BJ, Gottdiener JS, Epstein SE. Patterns and significance of the distribution of left ventricular hypertrophy in hypertrophic cardiomyopathy: A wide-angle, two-dimensional echocardiographic study of 125 patients. Am J Cardiol 1981; 48: 418-428.

22. Okawa M, Kitaoka H, Matsumura Y, Kubo T, Yamasaki N, Furuno $\mathrm{T}$, et al. Functional assessment by myocardial performance index (Tei Index) correlates with plasma brain natriuretic peptide concentration in patients with hypertrophic cardiomyopathy. Circ J 2005; 69: $951-957$.

23. Klues HG, Schiffers A, Maron BJ. Phenotypic spectrum and patterns of left ventricular hypertrophy in hypertrophic cardiomyopathy: Morphologic observations and significance as assessed by twodimensional echocardiography in 600 patients. $J$ Am Coll Cardiol 1995; 26: 1699-1708.

24. Hirota T, Kitaoka H, Kubo T, Okawa M, Furuno T, Doi YL. Morphologic characteristics of hypertrophic cardiomyopathy of the elderly with cardiac myosin-binding protein $\mathrm{C}$ gene mutations. Circ $J$ 2006; 70: $875-879$.

25. Elliott PM, Poloniecki J, Dickie S, Sharma S, Monserrat L, Varnava A, et al. Sudden death in hypertrophic cardiomyopathy: Identification of high risk patients. J Am Coll Cardiol 2000; 36: 2212-2218.

26. Olivotto I, Gistri R, Petrone P, Pedemonte E, Vargiu D, Cecchi F. Maximal left ventricular thickness and risk of sudden death in patients with hypertrophic cardiomyopathy. J Am Coll Cardiol 2003; 41: 315-321.

27. Maron BJ, Olivotto I, Spirito P, Casey SA, Bellone P, Gohman TE, et al. Epidemiology of hypertrophic cardiomyopathy-related death. Circulation 2002; 102: 858-864. 
28. Nihoyannopoulos P, Karatasakis G, Frenneaux M, McKenna WJ, Oakley CM. Diastolic function in hypertrophic cardiomyopathy: Relation to exercise capacity. J Am Coll Cardiol 1992; 19: 536-540.

29. Briguori C, Betocchi S, Romana M, Manganelli F, Losi MA, Ciampi $\mathrm{Q}$, et al. Excesise capacity in hypertrophic cardiomyopathy depends on left ventricular diastolic function. Am J Cardiol 1999; 84: 309 315 .

30. Kato T, Noda A, Izawa H, Nishizawa T, Somura F, Yamada A, et al. Myocardial velosity gradient as a noninvasively determined index of left ventricular diastolic dysfunction in patients with hypertrophic cardiomyopathy. J Am Coll Cardiol 2003; 42: 278-285.

31. Matsumura Y, Elloott PM, Virdee MS, Sorajja P, Doi Y, McKenna WJ. Left ventricular diastolic function assessed using Doppler tissue imaging in patients with hypertrophic cardiomyopathy: Relation to symptoms and exercise capacity. Heart 2002; 87: 247-251.

32. Olivotto I, Cecchi F, Casey SA, Dolara A, Traverse JH, Maron BJ. Impact of atrial fibrillation on the clinical course of hypertrophic cardiomyopathy. Circulation 2001; 104: 2517-2524. 